\title{
El papel de los sistemas regionales de innovación en ciudades región globales
}

The role of regional innovation systems in cities Global regions Le rôle des systèmes regionaux d'innovation dans des villes région globales

Henry Caicedo Asprilla hecaiced66@gmail.com Calle 4b No. 36-0o, Edificio 124 Universidad del valle, Sede San Fernando, Cali-Colombia

Estudiante del Doctorado en Economía y Gestión de la Innovación en la Universidad Autónoma de Madrid. Máster de Ciencias de la Organización de la Universidad del Valle. Profesor de Comercio Internacional y Economía del

Cambio Tecnológico en la Universidad del Valle.

Artículo Tipo: de revisión Según Clasificación Colciencias

Fecha de recepción: 10 de Febrero de 2011 Fecha de corrección: 03 de junio de 2011 Fecha de aprobación: 22 de junio de 2011

\section{Resumen}

Este documento es el resultado de una revisión de la literatura de los trabajos que intentan buscar el nexo entre los sistemas regionales de innovación y desarrollo de las ciudades región globales, y su propósito es mostrar que existe una complementariedad entre estos dos marcos de análisis y la pertinencia que tiene esta en la construcción de la política del desarrollo regional y urbano. Del análisis de los trabajos sobre este par de conceptos se advierte que la falta de una definición universalmente aceptada de región es el principal obstáculo para lograr integrarlos en las agendas de política regional; no obstante las investigaciones evidencian que los spillovers, los servicios intensivos en conocimiento y el diseño de política científica y tecnológica son los mecanismos a través de los cuales el conocimiento y la innovación se integran en el desarrollo regional. En esta perspectiva de análisis se concluye que las ciudades región globales como New York, Londres, Tokyo, Madrid, Milán, Sydney, entre otras son los principales nodos de comercio debido al uso intensivo que hacen del conocimiento.

Palabras clave: desarrollo regional, sistemas de innovación, spillovers, servicios intensivos en conocimiento.

Clasificación JEL: R58, 031, 032, L86, 
The role of regional innovation systems in cities Global regions

El papel de los sistemas regionales de innovación en ciudades región globales

\section{Le rôle des systèmes regionaux d'innovation dans des villes région globales}

\author{
El papel de los sistemas regionales de \\ innovación en ciudades región globales \\ The role of regional innovation systems \\ in cities Global regions
}

\begin{abstract}
This document is the result of a literature review of works trying to find the link between regional innovation systems and development of global city regions, and its purpose is to show that exist a complementarity between these two kinds of analysis and the relevance this has in the construction of regional policy and urban development. The analysis of the work on this pair of concepts warn the lack of a universally accepted definition of region is the main obstacle to integration into the regional political agendas, yet investigations show that spillovers, knowledgeintensive services and design of science and technology policy are the mechanisms through which knowledge and innovation are part of regional development. In this perspective it is concluded that global city regions such as New York, London, Tokyo, Madrid, Milan, Sydney, among others, are the main nodes of trade because they make intensive use of knowledge.
\end{abstract}

Keywords: regional development, innovation systems, spillovers, knowledge intensive services.

JEL code: R58, 031, 032, L86

\section{Résumée}

Ce document est le résultat d'une révision de la littérature des travaux qui visent à identifier la liaison entre les systèmes régionaux d'innovation et de développement des villes région globales ; et son objectif est celui de montrer qu'il existe une complémentarité entre ces deux cadres d'analyse, et la pertinence que celle-ci a sur la construction de la politique de développement régional et urbain. L'analyse des travaux sur ces deux concepts, permet d'affirmer que le manque d'une définition de région universellement acceptée constitue le principal obstacle pour réussir à les intégrer sur les sujets de politique régionale ; mais les recherches montrent que les spillovers, les services intensifs en connaissance et la conception d'une politique scientifique et technologique, sont les mécanismes à travers lesquels la connaissance et l'innovation s'intègrent dans le développement régional. Sur cette perspective d'analyse on peut conclure que les villes région globales comme New York, Londres, Tokyo, Madrid, Milan, Sydney parmi d'autres, sont les principaux nœuds de commerce grâce à l'usage intensif qu'elles font de la connaissance.

Mots clef: développement régional, systèmes d'innovation, spillovers, services intensifs en connaissance.

Classement JRL: R58, 031, 032, L86 


\section{El papel de los sistemas regionales de innovación en ciudades región globales}

\section{Introducción}

En la era de la globalización, las regiones se han venido consolidando como los ámbitos territoriales donde se concentra la aglomeración de personas y empresas, (Vásquez, 1999). Existe un conjunto de regiones que han aplicado una estrategia de desarrollo y de diferenciación, que les ha permitido transformarse en los principales centros mundiales de flujos de inversión, información y servicios; el resultado de esta dinámica de diferenciación es un orden jerárquico de ciudades en el que se destacan Londres, New York y Tokio como los principales nodos de comercio (Sassen, 2001); seguidas por regiones como Madrid, Milán y Shangai como de segundo orden (Taylor,2004); en un tercer renglón se hallan ciudades como Bogotá, Buenos Aires y Sao Paulo (Sassen, 2007); mientras que ciudades como Cali, Medellín y otras regiones colombianas y latinoamericanas apenas si logran engancharse en los flujos de comercio por lo que no aparecen en los rankings mundiales de ciudades globales (Cepal, 2009).

La identificación de una jerarquía de regiones que lideran el comercio mundial en un mundo cada vez más globalizado en el que se ha proclamado la muerte de la geografía (Morgan, 2004) ha suscitado un interés en los científicos sociales por entender el por qué, a medida que se profundiza en la globalización, la producción de bienes y servicios, la localización de la inversión y la toma de decisiones sobre la dinámica de los flujos de capital financieros están más concentrados en lugares particulares (Sassen, 2001; Scott et al. 2001).

Se han identificado al menos dos tipos de respuestas a esta cuestión; la primera proviene del desarrollo regional, en esta se agrupan economistas, geógrafos y sociólogos que tienen a la región y a lo urbano como objeto de estudio (Friedmann y Wolff, 1982; Sassen, 1995; Sassen, 2001; Castells y Hall, 1994; Castells, 2000; Scott et al. 2001). En la segunda línea de respuesta están los teóricos de la innovación quienes colocan en el centro del análisis al cambio tecnológico y como herramienta de política a los Sistemas Regionales de Innovación (SRI), (Florida 1995; Cooke, 1998; Morgan 1997; Edquist y Johnson 1997; Cooke, 1997; Lundvall 1998).
El propósito de este trabajo es, a partir de una revisión de la literatura, establecer la complementariedad entre el marco analítico de las ciudades región globales (CRG) y los SRI, con el fin de identificar los mecanismos de transferencia de la innovación y el conocimiento, a los procesos de aglomeración, accesibilidad, interacción espacial, competitividad, sostenibilidad y gobernabilidad de una ciudad región.

Los objetivos del trabajo son: a partir de una revisión de la literatura, identificar los mecanismos a través de los cuales el conocimiento se transfiere desde los SRI a los componentes del desarrollo regional de las CRG. A partir de la identificación de algunas experiencias de territorios, determinar el papel que ha tenido la innovación al posicionamiento de estos como CRG de segundo orden.

Este trabajo se divide en 4 secciones: la primera es la introducción, en la segunda se describen los marcos de análisis de las CRG y de los SRI, en la tercera se discute la complementariedad de los dos marcos de análisis, como componentes de la política de desarrollo regional; y se finaliza con un conjunto de conclusiones y recomendaciones.

\section{Los marcos de análisis de la ciudad región global y los sistemas regionales de innovación}

\subsection{La Ciudad Región Global}

El concepto de CRG, fue introducido a la agenda de investigación de geógrafos y economistas por Scott, Agnew, Soja y Storper, en el 2001, en un libro titulado: Global City Regions: Trend, Theory, Policy; se fundamenta en dos ideas previas: el de ciudad mundial de Friedmann y Wolff (1982) y el de ciudad global de Sassen (1995, 2001).

La noción de CRG se preocupa por cinco cuestiones claves: ¿Por qué las ciudades están creciendo tan rápidamente?, ¿Cómo han respondido ante la globalización las ciudades y regiones ante la nueva organización económica y social?, ¿Pueden las áreas metropolitanas de los países en desarrollo aprovechar los beneficios de la globalización incorporando nuevas ventajas com- 
petitivas? ¿Cuáles son las tareas de gobernanza a que se enfrentan las CRG?, ¿Cómo las nociones tradicionales de democracia y ciudadanía, están siendo cuestionadas con la aparición de las CRG? (Scott et al. 2001; Soja, 2005). Este trabajo se preocupa por las tres primeras en el sentido que pretende determinar el papel de la innovación en el desarrollo, formación y diferencias de las principales áreas metropolitanas a nivel mundial.

Las respuestas a estas preguntas se constituyen en las preocupaciones del programa de investigación de CRG; aquí se parte del reconocimiento de que en las últimas tres décadas las ciudades han experimentado un cambio en su organización espacial; entonces los temas de industrialización, urbanización y desarrollo económico y social, están siendo afectados profundamente por la globalización, con el agravante que los resultados son asimétricos entre los territorios (Soja, 2005).

En el programa de investigación de las CRG según Scott et al. (2001), Scott y Storper, (2003) y Soja (2005), una región es el resultado de las relaciones y acuerdos a que arriba el conjunto de entidades administrativas como municipios, provincias y comunidades que configuran un área metropolitana o de influencia económica en un territorio, que tienen como objetivo facilitar la formación de redes, alianzas, asociaciones, a nivel macro, meso y microeconómico, para responder a las amenazas y oportunidades que ofrece la globalización.

Note que esta idea de región se ajusta a la teoría de la economía de los costos de transacción de Coase (1937) y Williamson (1989): Una región se conformará bajo un área metropolitana o de influencia, hasta donde los costos de transacción que le impliquen a los agentes los procesos de vivir, trabajar y divertirse sean menores que llevar acabo estos procesos sin acuerdos, alianzas, fusiones y redes (Scott et al. 2001; Scott y Storper, 2003; Soja, 2005).

A partir de este concepto de región, Scott et al. (2001) pretenden explicar la dinámica mundial de los territorios; según este autor con la noción de CRG se puede analizar el desarrollo de ciudades como Londres, New york y Tokio, hasta justificar el por qué son las ciudades mas importantes del planeta en el sentido de Friedmann y Wolff (1982); a la vez explica el por qué estas ciudades basan el triunfo de sus economías en el sector servicios en la perspectiva de Sassen (2001).
La importancia del concepto de CRG es que, además de explicar a las ciudades globales de Sassen $(2001,2007)$ este puede dar cuenta del desarrollo de regiones como Singapur, Hong Kong, Silicon Valley, la ruta 128, regiones que se han caracterizado por transformar los procesos industriales hacia otros de mayor contenido tecnológico y que en la literatura se denominan regiones creativas o innovadoras (Florida 1995); al igual que puede explicar el desarrollo de regiones como Detroit y Cleveland en los EEUU, y las del antiguo bloque de Europa oriental, regiones que perdieron dinamismo industrial a finales de los 80 del siglo $\mathrm{XX}$, y que hoy están recomponiendo el proceso de industrialización y que se denominan como nuevos espacios industriales (Storper 1998).

Dada la definición de CRG y del tipo de territorios a que se aplica, es necesario identificar las características de esta clase de territorios y qué los hace diferentes de los demás; con ese fin es necesario precisar el núcleo central de este concepto. En este subyace la hipótesis central que la formación de una CRG, entraña tres procesos intrínsecamente unidos: el de la proximidad e interacción espacial y el de la competitividad y sostenibilidad (Scott et al. 2001; Scott y Storper, 2003; Soja 2005). A partir de este núcleo se derivan las características de las CRGs.

La primera se deriva del principio de proximidad; esta comprende tres procesos: cercanía, interacción espacial y conectividad o accesibilidad (Andersson y Karlsson, 2004; Morgan, 2004; Morgan, 1997; Florida, 1995; Cooke, 1998). La proximidad se favorece por las economías de escala, externas a la empresa e internas a la industria (Camagni, 2005).

En el contexto de la globalización, un rasgo sobresaliente de las CRGs es su capacidad de atraer trabajadores calificados capaces de ofrecer servicios intensivos en conocimiento (Sassen 2005). Al igual que generar servicios de salud, educación, por el alto índice de la esperanza de vida, por ofrecer condiciones que inducen a el crecimiento urbano (Soja, 2005; Scott y Storper, 2003).

Sassen (2001, 2005) y Taylor (2004), definen que una ciudad será más global entre mejor interconectada esté en la red de los flujos de información, financieros y del trabajo. Scott (2001), advierte que no sólo son las redes con el exterior del país las que definen una ciudad global o mundial. Este estatus lo logran ciudades 
que, además de interconectarse con el mundo, también establecen redes con los municipios, ciudades y territorios cercanos; estas alianzas son fundamentales para garantizar el suministro de los KIBS, un mercado de trabajo diferenciado, para tener reservas de suelo que permitan la diversificación de las actividades tanto de turismo, ocio, industriales, servicios, etc. (Scott et al. 2001; Scott y Storper, 2003; Soja, 2005).

El segundo conjunto de características, viene dado por la competitividad y sostenibilidad de los territorios. Este par de procesos están en la base económica de un territorio y comprende el comercio interregional e internacional, la competitividad o lugar que ocupa la ciudad en el concierto mundial de regiones, y sostenibilidad ambiental de los territorios (Camagni, 2005; Storper, 2003; Cooke, 1998).

Una CRG será más relevante entre mayor sea su posición en la jerarquía, ésta da cuenta de la posición que esta ocupa dentro del país (Camagni, 2005). En la era de la globalización, las jerarquías de territorios deben evaluarse en relación de la mundialización de la economía (Friedmann, 1986), porque el desarrollo de una ciudad global depende cada vez menos del país de origen y de las ciudades que lo componen mientras que dependen más del liderazgo en la red de los flujos de comercio, de capital y las funciones que cumple a nivel mundial (Sassen, 2005).

En relación a la competitividad y la base económica, una CRG, es considerada como locomotora de la economía e incubadora de empresas en el sentido que ofrecen más y mejores servicios empresariales que estimulan el nacimiento de nuevas firmas (Camagni, 2005; Storper, 1998); en este sentido una ciudad será más global entre más desarrollado sea el sector de los KIBS (Sassen, 2001).; pero estas no son las únicas condiciones, además están, la capacidad de albergar y permitir el desarrollo de clusters y/o distritos industriales en sectores capaces de integrar la tecnología a la producción (Scott y Storper, 2003).

En cuanto el medio ambiente, se admite que las ciudades son los agentes económicos que generan la mayor contaminación y demandan la mayor cantidad de energía en un ámbito geográfico determinado (Camagni, 2005); entonces se infiere que entre más globalizada sea una CRG, más dificultades tendrá para garantizar su sostenibilidad, debido a la proliferación de empresas, emigrantes y el crecimiento urbano incontrolado, porque estos fenómenos necesariamente presionan sobre los recursos naturales y los factores físicos del territorio (Storper, 2003; Soja, 2005).

Los procesos de proximidad e interacción espaciales, al igual que los de competitividad y sostenibilidad, requieren de un proceso de planificación (Camagni, 2005). Scott et al. (2001) argumentan que la CRG es el fruto de la necesidad de establecer nuevas formas de gobernanza para responder a la globalización. Los científicos sociales coinciden en que una ciudad región será más globalizada entre más y mejores alianzas pueda establecer. Una reflexión más general, en la que existe un relativo acuerdo, tiene que ver con la gobernanza necesaria, es decir, entre más global sea una ciudad región más actores participan en su desarrollo y más equilibrios políticos son establecidos para garantizar su funcionamiento; por ello se insiste en que la formación de CRG, es un proceso altamente influenciado por la política pública (Scott y Storper, 2003; Scott et al. 2001; Sassen, 2001; Sassen, 2005).

A manera de síntesis, se puede afirmar, que las CRG, alcanzan este estatus, gracias a que el desarrollo regional a nivel mundial, tiene las características de un desarrollo desequilibrado en el sentido de Hirschman (1961) y Myrdal (1959). Este par de autores, advertían que en un país existen regiones más prosperas que otras; argumento que es extrapolable al concierto mundial de ciudades, hoy a nivel mundial, existirán regiones que tendrán un proceso de crecimiento acumulativo y circular, mientras que otras experimentarán el efecto contrario, gracias a cuatro factores: un comercio basado en ventajas competitivas, un movimiento positivo de la mano de obra, un desplazamiento del capital, y la presencia de factores como la infraestructura que refuerzan las economías de aglomeración y localización.

En la actualidad los factores de Hirschman y Myrdal, se han reinterpretado en el concepto de base económica y competitividad, a través de las ideas de la formación de un mercado de trabajo, disponibilidad de factores intermedios, capacidad de absorción de tecnología (Krugman, 1992). Además de estos factores, a una región en un mundo cada vez más globalizado, la fortalece la disponibilidad de recursos no transferibles o idiosincráticos y la generación de interdependencias de activos no negociables que vuelven inmóviles algunos factores aparentemente de alta movilidad como el recurso humano (Morgan, 1997; Cooke, 1998). Entonces la interacción de estos factores 
pueden explicar el por qué unas regiones son más globales que otras, ya que en aquellas en que logran tomar la delantera, los factores generan unos efectos impulsores, mientras que en las regiones que se atrasan estos generan efectos de estancamiento (Myrdal 1959).

\subsection{Los Sistemas Regionales de Innovación}

La dinámica del desarrollo de las regiones también ha sido analizada por la economía y otras disciplinas que han tomado a la innovación como objeto de análisis. Los científicos de estas ciencias se han unido alrededor del concepto de SRI; el interés por las regiones, está asociado a la idea que la proximidad entre los agentes permite una mayor interacción y difusión del conocimiento tácito, que por definición, al no estar codificado, requiere de una relación cara a cara para incorporarlo a la innovación (Gertler, 2003; Morgan, 2004).

El énfasis de la dinámica y funcionamiento de los SRI está en el proceso de aprendizaje (Lundvall, 1992). Este proceso es el responsable de la creación de conocimiento, a partir de la transferencia de tecnología del exterior que facilita la difusión de las innovaciones (Lundvall, 1999). El aprendizaje permite que estos procesos ocurran a un costo menor, debido a la proximidad de los agentes (Cooke, 2004); por la mayor posibilidad de absorber las externalidades de la tecnología por los agentes (Jaffe. et al. 1993); a la vez por la posibilidad de combinar el aprendizaje derivado del uso del conocimiento explícito o basado en la ciencia y del conocimiento tácito basado en la experiencia (Lundvall, 2007).

Los SRI, están compuestos por tres esferas: un tejido empresarial, una infraestructura institucional y las interacciones que ocurren a los niveles macro, meso y micro (Freeman, 2001; Edquist y Johnson, 1997; Cooke, 1998; Andersson y Karlsson, 2006; Asheim e Isaksen, 2002). Son instituciones las leyes, políticas y planes de innovación, los convenios, acuerdos y contratos de transferencia de tecnología (Edquist y Johnson, 1997).

Por otra parte, las universidades, empresas, centros, laboratorios y organismos de intermediación financiera y de consultoría, son el grupo de organizaciones que suelen constituir un SRI (Edquist y Johnson, 1997) y (Cooke et. Al. 1997). Existe un relativo acuerdo en que los componentes de un SRI son cinco: el entorno institucional, el científico tecnológico, el productivo, el financiero y, por último, el de intermediación (Cooke, 2004).

Lundvall (2007), en el afán de definir y precisar el marco analítico de los sistemas de innovación, intenta trazar la frontera de este con respecto a los demás sistemas de la economía y sociedad; identifica que el tejido empresarial y las relaciones que este establece con las organizaciones científicas y tecnológicas se constituyen en el núcleo de un SRI; una vez definido el core, desde allí se pueden establecer los vínculos que el SRI crea con las demás esferas de la sociedad, como la del mercado de trabajo, el medio ambiente, sistema financiero, etc.; y, por su puesto, la contribución que la innovación hace al desarrollo en este caso, el regional.

En el desarrollo regional, los sistemas de innovación cumplen varios papeles o roles. Aquí interesan dos perspectivas, la institucional y la operacional. Desde la lógica institucional, un SRI tiene cuatro funciones: reducir la incertidumbre, proporcionar información, facilitar la cooperación y reducir los conflictos, por último, brindar incentivos para la innovación (Edquist y Johnson, 1997); mientras que a nivel operativo, el SRI, contribuye a facilitar las interacciones entre agentes, proporcionar recursos escasos para la innovación, y dinamizar la transferencia de tecnología y conocimiento desde y hacia el exterior a la región (Lundvall, 1997).

\section{Análisis de la relación entre las CRG y los SRI}

\subsection{Las Complementariedades Entre CRG y Los SRI}

El marco analítico de los SRI, evidencia problemas cuando se aplica a las regiones. Un primer cuestionamiento radica en que el resultado esperado del funcionamiento de los SRI, no es suficiente para explicar el conjunto de necesidades que implica el desarrollo regional, (Moulaert y Nussbaumer, 2005; Scott y Storper, 2003; Moulaert y Sekia, 2003). En cuanto los resultados de los SRI, Moulaert y Nussbaumer (2005), describen que los SRI, al igual que los modelos de desarrollo basados en la innovación, son limitados porque concentran y enfocan el análisis en aquellos aspectos asociados a la competitividad y crecimiento económico de las regiones, es como si la única función de la innovación y el conocimiento en la sociedad fuera mejorar la competitividad, y los temas de la calidad de vida, 
la sostenibilidad y la gobernanza estuvieran por fuera del alcance de la innovación, o, en el mejor de los casos, fueran un subproducto de la competitividad y el crecimiento.

Heidenreich, (2004) a partir de una revisión de 15 informes de SRI, de regiones localizadas en los cinco continentes, ha identificado un conjunto de problemas asociados a los SRI, que luego repercuten en el desarrollo de las regiones: la baja capacidad de innovación, el solapamiento y duplicación de esfuerzos entre las empresas e instituciones, la fragmentación y desarticulación de los agentes en cuanto el manejo y tratamiento de información; la brecha tecnológica entre la tecnología propia y las necesidades de las multinacionales; la hiperespecialización en una industria determinada con poca capacidad de diversificación; la brecha entre la investigación básica y la innovación tecnológica en las empresas y la dificultad de emprender nuevos negocios.

Una dificultad con que se enfrenta la relación entre CRG y SRI, es hallar la relación entre innovación y espacio (Morgan, 1997; Florida, 1995). El segundo obstáculo a tener en cuenta es el concepto de región que se maneja en ambas concepciones teóricas. Se advierte que desde el lado de la teoría de la innovación, no ha sido posible construir una definición de región universalmente válida. Se identifican al menos tres clases de definiciones:

Desde la lógica del aprendizaje. La región es el espacio geográfico donde opera un conjunto de instituciones e identidad cultural que permite compartir valores y por tanto, facilitan el aprendizaje y el intercambio del conocimiento tácito entre los agentes (Morgan, 1997; Florida, 1995).

En la perspectiva de las externalidades. Aquí una región se caracteriza por poseer un conjunto de factores que potencian la innovación: redes sociales, el desbordamiento del conocimiento, la proximidad de las organizaciones, las instituciones pertinentes, bajos costos de transacción de mercado entre proveedores y clientes; en conjunto, estos factores son los que en la literatura se conocen como interdependencias no negociadas. El aporte de estos factores es ayudar a generar toda clase de economías y permitir que unas regiones sean más innovadoras que otras (Storper, 1998).

En el marco de las agrupaciones industriales las regiones son definidas por las agrupaciones empresariales que poseen; estas pueden ser del tipo clusters (Porter, 1997) o Distritos industriales (Becattini 1979); se atribuye a las regiones la capacidad de crear agrupaciones de empresas capaces de actuar aglomeradas y, a partir de la proximidad entre ellas y los sistemas universitarios, financieros, de consultoría, etc, crear ventajas competitivas sustentables (Marcusen 1996).

A pesar de todos estos esfuerzos por definir la región, se advierte que en las definiciones existe cierta tautología en el sentido que a la región se le define por sus propiedades, características y logros. Así, la región existe porque posee cluster, facilita la innovación, genera externalidades; pero estas propiedades existen porque hay una zona geográfica donde se configuran, agrupaciones industriales, externalidades y aprendizaje (Moulaert y Sekia, 2003; Andersson y Karlsson, 2004; Iammarino, 2005; IRE, 2008; y Shearmur, 2008).

Llegados a este punto, se puede inferir que lo que ha dificultado la construcción del concepto de región puede deberse a que cada autor se concentra en un aspecto particular del fenómeno; de aquí que las visiones y definiciones se superpongan unas a otras generando más problemas que soluciones en el momento del diseño de políticas territoriales basadas en la innovación (Morgan, 2004; Andersson y Karlsson, 2004).

Con el fin de avanzar en la construcción de puentes que unan el desempeño de los SRI y la formación de las CRG, en este trabajo se propone que la idea de CRG de Scott et al, (2001); que, advierten que es dentro del área metropolitana 0 de influencia del territorio, donde cobran importancia: las externalidades o interdependencias no negociadas de Storper (1995), las agrupaciones industriales tipo clusters de Porter (1997), y distritos industriales de Becattini (1990), la proximidad geográfica que facilita el intercambio de conocimiento tácito de Morgan (2004), o el proceso de aprendizaje de Lundvall (2007), la formación de mercados de trabajo especializados (Krugman, 1992), la formación de una atmósfera industrial que induce la producción y la innovación (Marshall, 1920) tomando valor y adquiriendo significado para los agentes, porque se logra precisar un lugar en el que todas estas características operan y tienen un lugar de ocurrencia.

Se puede inferir que mientras el marco analítico de las CRG define el qué es una región, el de los SRI, define el cómo funcionan y alcanzan ventajas competitivas las regiones. Pero aquí 
surge la cuestión fundamental, ¿Cómo se articula el sistema de innovación al desarrollo regional en la era de la globalización? La respuesta a esta pregunta permite avanzar en la comprensión de cómo se crea, difunde y se concreta en servicios y productos el conocimiento en un territorio.

La complementariedad entre la innovación y el desarrollo regional, tropieza con el problema de la proximidad, en tanto que esta puede ser benéfica cuando estimula el aprendizaje o negativa cuando genera inercias institucionales (Langlois y Roberson 1995). Al respecto Boschma (2005) plantea que existen cinco tipos de proximidad que pueden incidir negativa o positivamente en el proceso de innovación: la proximidad geográfica, institucional, organizativa, cognitiva y social; con el fin de superar esta dicotomía analítica, plantea un conjunto de mecanismos que juntos pueden ayudar a que la proximidad se constituya en un incentivo para el aprendizaje y la innovación.

Los trabajos que han avanzado en el tratamiento de este problema han desarrollado tres tipos de explicaciones, implícitamente complementarias; un primer grupo de trabajos se ha concentrado en el análisis de la generación de spillovers geográficos (Jaffe et al, 1993); un segundo grupo aborda el tema de los SRI como productores de servicios intensivos y avanzados en conocimientos (Muller y Zenker, 2001;); el tercer conjunto de trabajos se concentra en el diseño de la política que evalúa como puede integrarse el sistema de innovación a los demás sistemas del territorio (Lundvall 2007).

1. La prueba de la relación positiva entre innovación y crecimiento regional a partir de la generación de los spillovers, ha sido motivo de múltiples trabajos:

a. Spillovers derivados de la movilidad del trabajo. Krugman (1991), mostró que las regiones más competitivas, son aquellas que poseen un mercado de trabajo especializado; se generan spillovers derivados de la movilidad de la mano de obra entre empresas, cuando los trabajadores cambian de empresa buscando mejores salarios. El proceso más estudiado es el de la movilidad de los trabajadores de las empresas multinacionales a las locales (Krugman, 1991; 1992) y hoy se acepta el movimiento en el sentido contrario de spillovers que ocurre cuando las multinacionales también se benefician de los conocimientos de los trabajadores de las empresas locales (Yang et al. 2008). b. Spillovers asociados a las agrupaciones empresariales. La segunda forma de difusión de conocimiento a través de los spillovers está asociada con la conformación de agrupaciones industriales tipo clusters (Porter, 1997) o distritos industriales o tejidos industriales, (Krugman, 1992). Las redes empresariales generan spillovers de múltiples maneras: la creación de canales de transferencia de tecnologías, estimula la difusión de nuevas formas organizativas de las empresas, impulsan a las empresas locales a adoptar estrategias de mejoramiento de calidad de los proveedores (Driffield y Love, 2005).

c. La tercera forma de spillovers se manifiesta a través de lo que Krugman (1992) denomina ósmosis tecnológica. Para mostrar que existía una concentración del conocimiento y la actividad innovadora en las regiones, Jaffe et al. (1993), probaron que la probabilidad de que una patente sea citada en la región o Estado de origen, es mayor que la probabilidad de que sea citada en otras regiones de los EEUU. Igualmente Audretsch y Feldman 2006, a partir de una función de producción de conocimiento mostraron que el desarrollo de esta está altamente correlacionado con factores tales como externalidades positivas, espíritu empresarial, generación de capital de riesgo, codificación del conocimiento y disponibilidad de habilidades y destrezas de los trabajadores.

2. En la literatura de la economía de la innovación y regional, se está evaluando el aporte que al crecimiento hacen la producción de servicios avanzados en conocimiento a la competitividad de las regiones (Vence y González, 2002; Muller y Zenker, 2001). Se agrupa bajo esta clase de servicios los llamados KIBS, que en esencia son de dos clases: los servicios profesionales tradicionales como consultorías, auditorías, contabilidad etc.; y algunos de bajo contenido tecnológico como la limpieza, alquiler inmobiliaria, reparación de máquinas y un largo etc.; la segunda clase de KIBS la constituyen las actividades de alto contenido tecnológico como la telemática, ingeniería de software, las pruebas de laboratorios, ensayos entre otros (Miles, 2005).

Muller y Zenker (2001) han identificado que los KIBS, tienen dos funciones en los SRI, actúan como el conocimiento externo que llega a las empresas innovadoras; por otra parte, esta clase de servicios son también el resultado de empresas que 
contribuyen a la generación de bienes y servicios de los demás componentes del sistema como un todo; a través de estas dos vías el conocimiento y la innovación se difunden a través del territorio y contribuyen a su desarrollo. El reconocimiento de este fenómeno es el que está en el centro de los programas de ciudades globales de Sassen (2001) y el de CRG, de Scott et al. (2001).

3. Para que los spillovers y los KIBS, actúen como mecanismos de transmisión de conocimiento, se requiere de una institucionalidad y diseño de política adecuado; la secretaría de las regiones innovadoras de Europa IRE (2008) ha identificado seis retos a vencer para lograr que la innovación llegue al desarrollo regional:

a. Interacción, deben facilitarse las condiciones para que los resultados de la innovación se difundan por el ámbito territorial.

b. Buena coordinación, aquí el problema es hacer compatibles las necesidades sociales y económicas de la región y los esfuerzos en investigación básica y aplicada.

c. Orientación y construcción de visión, aquí el reto es construir un escenario donde los problemas productivos sean compatibles con los de los científicos.

d. Estratégico, el problema a resolver es lograr introducir a la agenda de los investigadores, la generación de soluciones a la producción de servicios como la educación, salud, recreación, culturales, etc.

e. El uso de instrumentos, la barrera a derribar es el diseño de una institucionalidad científica que piense cómo hacer del territorio una región accesible, aglomerada, sostenible y económicamente viable.

f. Gobernanza multinivel y multicapa, el obstáculo a vencer es como facilitar las alianzas entre el estado, empresas y universidades en proyectos que tengan como ámbito de acción a la región.

Para resolver todos estos problemas, desde la geografía de la innovación, la idea de distancia cognitiva como mecanismo intangible que une a los agentes dentro de las economías de aglomeración, es un elemento clave para entender por qué unas regiones han podido superar con menos obstáculos estos retos que otras; Álvarez (2010), plantea que la distancia cognitiva es el elemento que une a las empresas en las agrupaciones industriales tipo clusters o distritos industriales, a su vez, esta idea unida a los cinco tipo de proximidades de Boschma, contribuyen a la comprensión del por qué unas regiones pueden articular más el conocimiento al desarrollo que otras con similar cantidad de recursos tangibles e intangibles.

\subsection{La relación empírica entre el desarrollo de Las CRG y los SRI}

Los trabajos que han evaluado empíricamente la relación entre los SRI y el desarrollo regional, se pueden clasificar en dos grupos. Los que evalúan la aglomeración, accesibilidad y conectividad de empresas y personas y su impacto en la innovación; por otro lado están los trabajos que analizan la contribución que la innovación hace a la base económica y sostenibilidad del territorio.

Existen múltiples trabajos que han contrastado la relación entre innovación y los componentes de la proximidad. Uno, especialmente relevante, es el de Johansson y Forslund (2006); estos autores muestran mediante un análisis a tres regiones de Alemania, en donde las empresas tienden a agruparse en función de las economías de escala y de urbanización, la fuente de estas economías está asociada a la disponibilidad de materias primas, personas y otras empresas que se localizaban verticalmente; también probaron que el conocimiento y las probabilidades de lograr una innovación determinan la aglomeración de empresas.

Un segundo trabajo destacable en la línea de la relación entre innovación y aglomeración de personas, es el realizado por Sharpe y Martínez (2006), las autoras probaron que el mercado de trabajo en el Área metropolitana de Sídney se distribuye en función del tipo de servicios que brindaban los trabajadores, en al menos tres áreas: en el área del sur se localizan los trabajadores que brindan servicios rutinarios asociados a la manufactura; en el área del centro se ubican los trabajadores que brindan servicios personales, maestros, médicos, abogados, etc; en el área del norte se agrupan los trabajadores del futuro, aquellos capaces de analizar y brindar soluciones a problemas, estos venden sus servicios a todo el mundo, y son los que le están permitiendo a Sidney ser una ciudad región global.

El tema de la accesibilidad, como bien argumentan Andersson y Karlsson (2004), ha sido una 
relación poco explorada, y el análisis se ha realizado en el sentido contrario, es decir, el impacto que tiene la accesibilidad en la innovación; esfuerzos similares se pueden identificar en Morgan (2004), Florida (2002); Cooke (2001). Esta hipótesis ha sido abordada desde varias perspectivas:

a. Un primer tipo de análisis es el que relaciona a la innovación con la distancia y tiempo máximo que los agentes están dispuestos a recorrer para intercambiar información y realizar negocios. Andersson y Karlsson (2004) mostraron que en las regiones de Suecia, los agentes realizaban intercambios de información y de negocios cara a cara, en un radio de acción de $60 \mathrm{Km}$ y un tiempo de 45 minutos.

b. Otra manera de abordar esta hipótesis es a través del análisis de la relación entre la geografía y la concentración del conocimiento. Autores como Morgan (2004); Florida (2002); Cooke (2004) Gertler (2003), han mostrado que la proximidad entre los agentes favorece la innovación gracias a la posibilidad del intercambio de conocimiento tácito que, por definición, es no codificable y, por tanto, no es transmisible a largas distancias, al contrario requiere de las relaciones cara a cara para ser económicamente útil.

c. La tercera forma de probar la hipótesis de relación entre la aglomeración, conectividad e innovación, es a través de la generación de servicios avanzados en conocimiento de una región global. Sassen (2001), ha planteado que las ciudades globales adquieren este estatus cuando logran hacer compatibles al menos tres procesos: la generación de servicios de conocimiento, ser lugar de innovaciones, lugares con liderazgo mundial en la economía y ser lugar de inversión extranjera directa; los estudios sobre ciudades región globales han mostrado que en esta clase de territorios, más del $60 \%$ de su economía la compone el sector servicios avanzados en conocimiento (Scott et al. 2001; Soja, 2005).

Un segundo grupo de trabajos ha mostrado que existe una relación positiva entre los esfuerzos de innovación realizados desde el SRI, y la base económica, la sostenibilidad y la jerarquía de la región a nivel mundial. Esta hipótesis es tal vez la más demostrada en la literatura de la innovación, (Moulaert y Sekia, 2003; Moulaert y Nussbaumer, 2005); estos trabajos se agrupan bajo el nombre de Modelos de Innovación Terri- torial MIT; entran en esta categoría los estudios de clusters de (Porter, 1990; 1997), los Milieux Innovateurs de Camagni (1991); los trabajos sobre distritos industriales de Becattini (1979) y nuevos espacios industriales de Storper, (1997).

Aunque estos estudios tienen a la aglomeración, conectividad y accesibilidad como elementos de explicación, o condiciones necesarias para la base económica y sostenibilidad de la región, es cierto que las hipótesis a demostrar se concentran en la relación entre innovación y formación de agrupaciones empresariales y el resultado de estas en el incremento de la competitividad de la región, también avanzan hasta mostrar la relación entre innovación y uso de los recursos usados en la producción, y cada vez más se evalúa el resultado global de la competitividad y sostenibilidad en la jerarquía o posición de la región en el concierto mundial de ciudades.

A partir de los estudios seminales, se ha desarrollado una industria de trabajos que prueban las hipótesis de relación positiva entre la innovación y el desarrollo regional: en esta línea de investigación se ha mostrado a través de la simulación en las regiones alemanas que existe una relación positiva entre los esfuerzos de innovación desde los SRI y el crecimiento de las diferentes ramas productivas; lo relevante de estos trabajos radica en que logran evaluar esta relación mediante la operación de estímulos de demanda, originados por gastos del gobierno o exportaciones, y desencadenar crecimiento al conjunto de la economía, de esta manera se absorben los gastos de la innovación vía amplitud del mercado.

En esta perspectiva Markusen (1996), rechaza la idea básica de distrito industrial en la versión italiana de (Becattini, 1979), y plantea que más bien las agrupaciones industriales se asocian a características del desarrollo de las regiones e identifica cuatro tipos de distritos: el Marshalliano, el Hub-and-Spoke: los jalonados por una empresa dominante; State-anchored: los dinamizados por empresas orientadas a la exportación y Satellite Platform impulsados por el sector público.

La relación entre innovación y crecimiento se ha evaluado a través de la existencia de un mercado de trabajo (Krugman, 1992), a partir de esta premisa, se ha desarrollado un conjunto de trabajos que analizan cómo ocurre esta relación, en esta dirección el trabajo de Stolarick et al. (2005), a partir de un estudio del área metropolitana de Montreal, demuestran que la densidad 
y diversidad del mercado de trabajo creativo constituido por científicos, ingenieros y artistas, guarda una relación positiva con la innovación y el crecimiento regional. Asheim e Isaksen (2002), probaron a través de un estudio a tres regiones de Noruega, que la formación de Clusters dependía de la disponibilidad de recursos locales y la formación de un capital humano formado en el lugar, esta combinación le da un carácter de inmovilidad a los recursos y una diferenciación sobre la cual descansa la competitividad y el crecimiento de las regiones.

Una vertiente relevante de investigación entre los SRI y la formación de las CRG, se halla en el analisis de la planificación y diseño de la política. Recuérdese que el espíritu del concepto de sistemas de innovación es tratar de devolverle a la política pública el protagonismo perdido con el auge de la globalización y el Consenso de Washington. Por su parte Camagni (2005), propone en el manual de economía regional, que la planificación urbana y el diseño de política pública que coordine los intereses de los grupos de interés de la región, cada vez más es un principio de desarrollo regional tan importante como el de aglomeración, accesibilidad y los demás.

En esa perspectiva Isaksen (2001), plantea que es una necesidad construir sistemas de innovación regionales a medida que la economía se globaliza, porque si globalización es sinónimo de internacionalización del capital, cada vez que las empresas multinacionales deciden modificar su estructura productiva, deciden la suerte de las regiones donde se localizan; por consiguiente los policy makers, deben tener un plan para poder lograr atraer inversión extranjera y poder resolver los problemas que surgen cuando ésta por algún motivo deja la localidad.

A partir de esta reflexión, Andersson y Karlsson (2004) mediante una revisión de la literatura, mostraron que los sistemas de innovación variaban con el tamaño de las regiones, existían regiones pequeñas con empresas pequeñas y medianas y que por tanto requerían de un diseño de política diferente a los sistemas bien consolidados y que sólo estaban presentes en las regiones de gran tamaño. Por lo tanto, se hace necesario construir un diseño de política acorde a las características de la región, es decir, no existen recetas transmisibles de un lugar a otro sin probabilidad de fracaso.
En los manuales de economía regional y de ciudades globales, cada vez es más recurrente incluir como un capítulo obligado el tema de la sostenibilidad. Se recomienda que se haga uso de la innovación y el conocimiento como estrategias tratamiento del medio ambiente (Camagni 2005; Sassen 2001; Scott et al. 2001). La relación de SRI y sostenibilidad ha sido explorada por Lundvall y Johnson, (2002); pero como parte integral del desarrollo sostenible, a partir de la manera como la tecnología y la innovación actúan como ahorradores para hacer de las ciudades y regiones los principales focos de recursos naturales; no obstante se carece de estudios empíricos que hayan probado esta hipótesis; pero no hay duda que esta relación se ha tratado desde otras corrientes tales como el de la ecoinnovación, tecnologías limpias, etc., pero el tratamiento de esta cuestión escapa a los propósitos de este trabajo.

\section{Conclusiones}

Aquí se ha presentado los conceptos de CRG y de SRI, como dos elementos que hoy están en la agenda de la política urbana y regional. Las virtudes de este par de ideas, radican en que invitan a reflexionar sobre cómo hacer para que los territorios cada vez más sean espacios de convivencia y progreso en el que las personas y empresas encuentren elementos para mejorar la calidad de vida y competitividad. Pero también se ha mostrado que esta no es tarea fácil, pues son muchos retos que de orden político e institucional los que enfrenta una región para lograr ser territorio inteligente e innovador. De la revisión de la literatura se puede concluir que si bien no es tarea fácil, existen procesos que pueden ser imitados, con éxito, por ejemplo, es sorprendente como Madrid, pasó a ser una ciudad de segundo orden en menos de 15 años; de aquí que en la agenda de investigación de las CRG, está abierto el debate, sobre cómo lograr que cada vez más regiones se enganchen con éxito en el concierto mundial de ciudades.

De la revisión de la literatura se puede afirmar que es posible hallar una relación directa entre el uso del conocimiento y el mejoramiento en los patrones de desarrollo regional; la sostenibilidad ambiental, la competitividad y cohesión social. Los hallazgos de estudios aquí citados muestran que entre más global sea una ciudad, mayor es la intensidad con que usa el conocimiento y genera spillovers, produce más y mejores servicios intensivos en conocimiento y mejor diseñada tiene la política científica y tecnológica. De aquí que 
futuras líneas de investigación se abren: ¿Qué deben hacer los territorios que deseen ser globales para engancharse con éxito en la era de la globalización? ¿Cómo hacer del conocimiento un factor de producción que ayude a los territorios a mejorar sus potencialidades y maximizar el uso de los recursos? Estas y otras preguntas quedan abiertas cuando se integra al desarrollo regional el conocimiento y las infraestructuras de innovación como las de los SRI.

\section{Referencias}

Álvarez, F. (2006) "Economía de Aglomeración: La Distancia Cognitiva en la Creación De Una Empresa" En: knowledge management lab, dimeg, politecnico di bari en viale japigia 182, 70126 bari, italy.

Andersson, M. y Karlsson, C. (2004). The Role of Accessibility for Regional Innovation Systems. En: Karlsson, C., Flensburg, P. y Hörte, S.-Å. (Eds.). Knowledge Spillovers and Knowledge Management, Edward Elgar, Cheltenham (Forthcoming).

Andersson, M. y Karlsson, C. (2006). Regional Innovation Systems in Small and MediumSized Regions. En: Johanson, B, Karlsson, C. y Stough, R. (Eds), The Emergin Digital Economy, entrepreneurship, Clusters and Policy (55-81). Berlin: Springer-Verlag.

Asheim, B. e Isaksen, A. (2002). Regional Innovation Systems: The Integration of Local 'Sticky' and Global 'Ubiquitous' Knowledge. The Journal of Technology Transfer, 27(1), 77-86.

Leonard-Barton, D. (1995). Wellsprings of knowledge: Building and sustaining the sources of innovation. Boston MA: Harvard Business School Press.

Becattini, G. (1979). Dal 'settore' industriale al 'distretto' industriale. Alcune considerazioni sull'unità d'indagine dell'economia industriale. Rivista di economia e politica industriale, 5(1), 7-21.

Boschma, R., (2005). Proximity and innovation: a critical assessment, Regional Studies, Vol. 39, (1): 61- 75 .

Camagni, R. (1991). Local 'milieu', uncertainty and innovation networks: towards a new dynamic theory of economic space. En: Camagni, R.
(Eds). Innovation Networks: Spatial Perspectives (121-142). London: Belhaven.

Camagni, R. (2005). Economía Urbana. (1ae ed. en español, pp. 1-20). Barcelona: Antoni Bosch.

Castells, M. y Hall, P (1994). Tecnópolis del mundo, la formación de los complejos industriales del siglo XXI. (1를., pp. 209-272). Madrid: Alianza editorial.

Castells, M. (2000, febrero). La ciudad de la nueva economía. Documento presentado en el Salón de Ciento del ayuntamiento de Barcelona, Barcelona, España.

CEPAL. (2009), Panorama social de América Latina 2008. Santiago de Chile: Publicación de las Naciones Unidas, № de venta: S.08.II.G.89.

Recuperado el 22 de noviembre de, 2010, de http:// www.eclac.org/publicaciones/xml/2/34732/ PSE2008_Versioncompleta.PDF

Coase, R.H. (1937). The Nature of the Firm. Economica, 4(16), 386-405.

Cooke, P., Gómez, M., Etxebarria, G. (1997). Regional innovation systems: Institutional and organisational dimensions. Research Policy, 26, 475-491

Cooke, P. y Morgan, K. (1998). The associational economy. Firms, regions and innovations. Oxford: Oxford University Press.

Cooke, P. (2001). Regional Innovation Systems, Clusters, and the Knowledge Economy. Industrial and Corporate Change, 10 (4), 945-974.

Cooke, P., (2004). Regional Innovation Systems - an evolutionary approach. En: Cooke, P., Heidenreich, M. y Braczyk, H-J. (Eds.), Regional innovation systems: the role of governance in a globalized world (1-20). Londres: Routledge.

Driffield, N., Love, J., (2005). Who gains from whom? Spillovers, competition and technology sourcing in the foreign-owned sector of uk manufacturing. Scottish Journal of Political Economy, 52(5), 663-686.

Dunning, J.H. (1998). Location and the Multinational Enterprise: A Neglected Factor. Journal of International Business Studies, 29 (1), 45-66. 
Edquist, C. y Johnson, B. (1997). Institutions and Organizations in Systems of Innovation. En: Edquist, C. (Eds.) Systems of innovation: technologies, institutions, and organizations (41-63). Londres: Routledge.

Florida, R. (1995). Toward the learning region. Futures, 27(5), 527-536.

Florida, R. (2002). The rise of the creative class: And how it's transforming work, leisure, and everyday life (Ed.). New York: Basic Civitas Books.

Freeman, C. (2001). Perspectiva histórica del "Sistema Nacional de Innovación”. En: Gómez, M. y Olazarán, M. (Eds.). Sistemas regionales de innovación (29-56). España: Universidad del País Vasco, Servicio de Publicaciones.

Friedmann, J. y Wolff, G. (1982). World city formation: an agenda for research and action. International Journal of Urban and Regional Research, 6(3), 309-344.

Friedmann, J. (1986). The World Cities Hypothesis. Development and Change, 17, 69-84.

Gertler, M.S. y Levitte, Y.M. (2003, junio). Local nodes in global networks: the geography of knowledge flows in biotechnology innovation. Documento presentado en el DRUID Summer Conference, Elsinore, Dinamarca.

Heidenreich, M. (2004). The Dilemmas of Regional Innovation Systems. En: H. Braczyk, P. Cooke and $M$. Heidenreich (Eds.), Regional Innovation Systems: The Role of Governance in a globalized World (363-389). Londres, New York: Routledge.

Hirschman, A. O. (1958). The strategy of economic development. New Haven:Yale University Press.

IRE, (2008). Effective Regional Innovation Systems. Final report. Luxemburgo: IRE Working Group Effective Regional Innovation Systems, $71 \mathrm{p}$.

Isaksen, A. (2001). Building Regional Innovation Systems: Is endogenous industrial development possible in the global economy? Canadian Journal of Regional Science, Vol. 24.
Jaffe, A.B., Trajtenberg, M., Henderson, R. (1993). Geographic localization of knowledge spillovers as Evidenced by Patent Citations. The Quaterly Journal of Economics

Johansson, B. y Forslund, U. (2006). The analysis of location, co-location and urbanisation economies. (Estocolmo: CESIS Electronic Working Paper Series). Recuperado el 29 de noviembre de 2010, de www.infra.kth.se/cesis/documents/WP67.pdf

Krugman, P. (1991). Increasing Returns and Economic Geography. The Journal of Political Economy, 99(3), 483-499.

Krugman, P. (1992). Geografía y Comercio (1ae ed., pp. 41-76). Barcelona: Antoni Bosch.

Lundvall, B.A. (1992). National Systems of Innovation: Towards a theory of innovation and interactive learning (Ed.). Londres: Pinter

Lundvall, B.A. (1998). Why study national systems and national styles of innovation?. Technology Analysis \& Strategic Management, 10(4), 403 $-422$.

Lundvall, B.A. (1999). La base del conocimiento y su producción. Ekonomiaz, 45, 14-37.

Lundvall, B. A. (2007). National innovation system - analytical concept and development tool. Industry and innovation, 14, 1; ABI/INFORM Global, pp. 95-119.

Marshall. A. (1920). Principios de Economía (3 $3^{\underline{a}}$ Ed en español. 223-232). Madrid: Aguilar.

Markusen, A. (1996) "Sticky places in slippery space: a typology of industrial districts". Economic Geography, 72(3): 293-313.

Miles, I. (2005). Innovation in Services. En: Fargerberg, J., Mowery, D. y Nelson, R. (Eds.) The Oxford Handbook of Innovation. (433-458). New York: Oxford University Press.

Myrdal, Gunnar. (1959) Teoría económica y regiones subdesarrolladas. México: Fondo de Cultura Económica, 1ra. ed.

Morgan, K. (1997). The Learning Region: Institutions, Innovation and Regional Renewal. Regional Studies, 31, 491-504. 
Morgan, K. (2004). The exaggerated death of geography: learning, proximity and territorial innovation systems. Journal of Economic Geography, 4(1), 3-21.

Moulaert, F. y Sekia, F. (2003), Territorial Innovation Models: A Critical Survey. Regional studies, 37(3), 289-302.

Moulaert, F y Nussbaumer, J. (2005). La Región Social. Más allá de la dinámica territorial de la economía del aprendizaje. Ekonomiaz. 58, 96-127.

Muller, E. y Zenker, A. (2001). Business services as actors of knowledge transformation: the role of KIBS in regional and national innovation systems. Research Policy, 30(9), 1501-1516.

Porter, M. E. (1997). Estrategia competitiva: técnicas para el análisis de los sectores industriales y de la competencia ( 1 a ed, pp.93-158). México D.F.: Compañía Editorial Continental.

Sassen, S. (1995. Marzo). The Global City: Place, production and the new centrality. Documento presentado en seventh National Conference of the Association of College and Research Libraries, en Pittsburgh, Estados Unidos.

Sassen, S. (2001). The global city: New York, London, Tokyo (2a ed., 127-361). Princeton University Press.

Sassen, S. (2005). The Global city: Introducing a concept. Brown journal of World Affairs, 11(2), 27-43.

Sassen, S. (2007). El reposicionamiento de las ciudades y regiones urbanas en una economía global: ampliando las opciones de políticas y gobernanza. Eure, 33(100), 9-34.

Scott, A. J., Agnew, J., Soja, E.W., y Storper, M. (2001) Global city regions. En Scott, A. (Eds.) Global city-regions: trends, theory, policy (1131) USA: Oxford University Press.

Scott, A. J. y Storper, M. (2003). Regions, Globalization, Development. Regional Studies, 37(6/7), 579-93.

Sharpe, S. y Martínez, C. (2006. Junio). Measuring regional knowledge resources: what do knowledge occupations have to offer? Documento presentado en The DRUID Summer
Conference 2006 on Knowledge, innovation and competitiveness: Dynamics of firms, networks, regions and institutions, en Copenhague, Dinamarca.

Shearmur, R. (2008). Neo-Regionalism and Spatial Analysis: Complementary Approaches to the Geography of Innovation? (Toronto: ISRN working paper). Recuperado el 24 de noviembre, de www.utoronto.ca/isrn/publications/WorkingPapers/Working08/Shearmuro8_neoregionalism.pdf

Soja, E.W. (2005). Algunas consideraciones sobre el concepto de ciudades región globales. Ekonomiaz, (58), 44-75.

Stolarick, K., Florida, R. y Musante, L. (2005). Montréal's capacity for creative connectivity: Outlook and opportunities. (Montreal, Q.C.: Working paper Catalytix). Recuperado el 13 de diciembre de 2010, de creativeclass.com/ rfcgdb/articles/montreals capacity.pdf

Storper, M. (1995). The Resurgence of Regional Economies, Ten Years Later. European Urban and Regional Studies, 2(3), 191-221.

Storper, M. (1998). Las economías regionales como activos relacionales. Ekonomiaz, 41, 10-45.

Vence, X. y González, M. (2002). Los servicios y la innovación. La nueva frontera regional en Europa. Economía Industrial, 347, 41-66.

Williamson, O. (1989). Las Instituciones Económicas del Capitalismo (1a ed. en español, pp. 26 52). Mexico D.F.: Fondo de Cultura Económica. 